\title{
ATYPICAL AXILLARY LYMPHADENOPATHY MIMICKING AN OCCULT BREAST CARCINOMA IN A PATIENT DIAGNOSED WITH SARCOIDOSIS: CASE REPORT
}

\author{
Paula Clarke , Carolina Nazareth Valadares¹, Douglas de Miranda Pires , Nayara Carvalho de Sá1 \\ 1Santa Casa de Misericórdia de Belo Horizonte - Belo Horizonte (MG), Brazil.
}

Introduction: Occult breast carcinoma is a rare presentation of breast cancer, with histological evidence of axillary lymph node involvement and clinical and radiological absence of malignant breast lesions. Its survival is similar to that of the usual presentation. The treatment consists of modified radical mastectomy or axillary drainage with breast irradiation, resulting in similar survival, associated with systemic therapy according to the staging. Neoadjuvant therapy should be considered in N2-3 axillary cases. Differential diagnoses of axillary lymphadenopathies include: non-granulomatous causes (reactive, lymphoma, metastatic carcinoma) and granulomatous causes (infectious - toxoplasmosis, tuberculosis, sarcoidosis, atypical mycobacteria). Objectives: To report the case of a patient who needed a differential diagnosis among the various causes of axillary lymphadenopathy. Methods: This is a literature review conducted in the PubMed database, using the keywords «granulomatous lymphadenitis», «breast sarcoidosis», «occult breast cancer». Inclusion and exclusion criteria were applied. Case report: V.F.S., female, 51 years old, was referred to an evaluation of axillary lymphadenopathy in May 2019. She was followed by the department of pulmonology due to mediastinal sarcoidosis since 2017. Physical examination indicated breasts without changes. Axillary lymph nodes had increased volume and were mobile and fibroelastic. Mammography revealed only axillary lymph nodes with bilaterally increased density, and the ultrasound showed the presence of atypical bilateral lymph nodes. Neither presented breast lesions. Axillary lymph node core biopsy was compatible with granulomatous lymphadenitis. This result corroborates the diagnosis of sarcoidosis affecting peripheral lymph nodes. The patient was referred back to the department of pulmonology, with no specific treatment since she is oligosymptomatic. Discussion: Despite the context of benign granulomatous disease, malignancy overlying the condition of sarcoidosis must be ruled out. The biopsy provided a safe and definitive diagnosis, excluding the possibility of occult breast carcinoma. The patient will continue to undergo breast cancer screening as indicated for her age and usual risk. Conclusion: In the presentation of axillary lymphadenopathy, the mastologist must know the various diagnoses to be considered. The most feared include lymphoma and carcinoma metastasis with occult primary site. A proper workup can determine the diagnosis and guide the appropriate treatment. 